\title{
The Present Situation and Developing Strategy of Zhuxianzhen New Year Woodprint's industrialization
}

\author{
Hongting Xiao \\ Huanghe Science \& Technology College \\ Zhenghzou, China
}

\begin{abstract}
Based on the disappointing present situation of Zhuxianzhen New Year woodprint, such as the lack of talented apprentices, the marketing disorder and outdated concept and so on, this paper focuses on the necessity and feasibility of its industrialization. It also explores the developing strategies for Zhuxianzhen New Year woodprint, including the realization of scientific planning and market operation, the construction of innovative team, the founding of creative industry park etc.
\end{abstract}

Keywords-Zhuxianzhen New Year woodprint; present situation; developing strategy

\section{INTRODUCTION}

New Year woodprint is one of the Han folk arts with profound history, in which Zhuxianzhen's is a distinctive essence of Chinese traditional folk art. Zhuxianzhen New Year woodprint has great value both of art collection and appreciation. It gets its name as it is mainly produced in Zhuxian Town, Henan Province. The place began to produce New Year pictures in Han Dynasties, and it prospered in Ming and Qing Dynasties. The themes of Zhuxianzhen woodprints mainly vary from portraits of martial and intellectual gods to portraits of spirits. The pictures printed in Zhuxianzhen are very popular due to their bright colors, exaggerated images, rough lines and full compositions. Famous writer Lu Xun once highly lauded the New Year picture produced in the place, saying: "The carving lines of Zhuxianzhen woodprints are very macho; it's not elaborately polished, very unlike those in other places. The woodprints are simple, with no extra adornment. The figure painting has very strong color, without being overbearing. It shows the strong local flavor which is typical in North China woodprints." However, with the developing of technology, it is hard for Zhuxianzhen woodprint to keep up with the capricious tastes of its viewers, which reveals some existing problems in its development.

\section{THE PRESENT SITUATION AND PROBLEMS IN THE INDUSTRIALIZATION PROCESS OF ZHUXIANZHEN NEW YEAR WOODPRINT}

First, it will be lost for the shortage of apprentice. Statistics show that most of the old generation craftsmen passed away, and only three old craftsmen are still alive. Nowadays, there are no more than ten craftsmen, and all of them are in their 50s. The shortage of apprentice makes Zhuxianzhen New Year woodprint in the danger of extinction.
Second, the existing workshops are small, lacking modern operating and marketing strategies. Investigations suggest that there are about 20 workshops producing and selling Zhuxianzhen New Year woodprints; most of them are located at countryside. Besides, the producers and managers are farmers; they have ancestral craftsmanship but lack modern marketing strategies. This becomes the second block for its industrialization.

Third, the operating concept of Zhuxianzhen New Year woodprint industry is backward because of the shortage of creative talents, from designers, craftsmen, painters, to industrial engineering technology personnel and research exploiters as well as lawyers and financial talents. In order to boom Zhuxianzhen New Year woodprint, modern operating concept and creative personnel should be brought into the industry.

The reasons for the problems above mainly exist in the following points: the value both of the Zhuxianzhen New Year woodprint and its producers has been ignored; the protecting strategies and promoting effort are far from enough; the clash between the traditional craftsmanship and advanced new technology still exists.

First, the value both of the Zhuxianzhen New Year woodprint and its producers has been ignored. In the course of Zhuxianzhen New Year woodprint history, lots of craftsmen with sound skills devote their lives to it. Unfortunately, they have to struggle to earn enough money for their family. What's worse, there are few apprentice craftsmen. The lack of effective protection on intellectual property right of folk arts makes the situation even more disappointing. In international market, the price of Zhuxianzhen New Year woodprint is too low to support and attract more practitioners, which may bring a vicious circle for the industry.

Next, the protecting strategies and promotion are far from enough. Not more than 60 Zhuxianzhen New Year woodprint models are still exist today, which are second and third Grade Cultural Relics of China. 6 of them were made in Ming Dynasty, 23 in Qing Dynasty, and 28 in the period of the Republic of China. In the protection of the Zhuxianzhen New Year woodprint, much more practical strategies should be implemented. What's more, the promotion effort from the government and relative authorities is also necessary. 
Last but not least, the clash between the traditional craftsmanship and advanced new technology still exists. From the past century, the impact of the popularization of modern printing technologies has brought a heavy blow to this traditional trade. As a result, the market demand for the woodprint New Year pictures shrinks rapidly, forcing many old workshops to close. Because of the depressing market, the producing quantity of Zhuxianzhen New Year Woodprints is so limited that it is hard for many loyal buyer or collectors to choose the satisfactory prints, which makes the market even more depressing.

\section{THE NECESSITY AND FEASIBILITY OF ZHUXIANZHEN NEW YEAR WOODPRINTS' INDUSTRIALIZATION}

Because of the depressing present situation and existing problems of Zhuxianzhen New Year woodprint, it is very necessary for us to protect and promote this valuable non-material cultural heritage. What's more, creative thinking and innovation should be advocated in the protection process.

\section{A. The necessity of Zhuxianzhen New Year woodprints' industrialization}

Firstly, it is beneficial to the establishment of cultural brand for the city. Some accomplishments have been achieved these years in the close unity of Zhuxianzhen New Year woodprint development and local urban construction. Zhuxianzhen is still the economy center in the zone though it is not as prosperous as it once was in Ming and Qing Dynasty. The local government has put massive investment into the construction of civil and cultural subjects, the building of highways, schools, museums, cultural centers, and the foundation of Zhuxianzhen Woodprint New Year Pictures Research Center. Under this background, it is necessary for us to accentuate the "spirit" and "shape" in Zhuxianzhen New Year woodprints, with creative new ideas in the design of innovative products and souvenirs, such as canvas bags, stickers and so on.

Secondly, it is beneficial to the local construction of distinctive cultural images. By the exploiting of local styles and customs, Zhuxianzhen New Year woodprint can be refashioned as an important component of urban culture brand. It also plays a significant role in tourism, because it can attract more folk art lovers and boost the prosperity of local service industries. In addition, it can attract more investment which will stimulate the development of local economic chain. The industrialization of Zhuxianzhen New Year woodprint is based on its cultural characteristics and connotation, which will improve the image and cultural connotation of Kaifeng, Henan, and the whole central area in China as well. The purpose of its industrialization is to boom the local economy; what's more important is to advocate creative thinking and innovation ideas.

\section{B. The feasibility of Zhuxianzhen New Year woodprints' industrialization}

Nowadays, the influence of creative industry to GDP cannot be neglected. Consensus has been reached about the significant role of innovation and cultural arts, which is not only a concept, but an explicit reflection of the massive economic benefit. The following part focuses on the feasibility of Zhuxianzhen New Year woodprints' industrialization.

Firstly, Zhuxianzhen (Zhuxian Town), as one of the five most important towns for prior development in Henan Province since 2001, has convenient transportation. Besides, it becomes well-known because of its native products, in which New Year woodprint and red paper are the most famous ones. From the record in Dongjingmenghualu, it is not hard to reflect the unprecedented prosperity of Jingdong (today's Kaifeng) New Year woodprint then.

Secondly, Zhuxianzhen has a rather profound culture. And the New Year woodprint here has more than 1000 years history; thus it is the forefather of many Chinese New Year pictures. For example, Yangliuqing New Year picture in Tianjin, Weifang New Year picture in Shandong and Taohuawu New Year picture in Jiangsu are originated from and influenced by it at some extent. In addition, it also sheds some light on the New Year pictures in Wuqiang Hebei, Foshan Guangdong, Mianzhu Sichuan, Zhangzhou Fujian and other places. The basic techniques for New Year pictures printing in Zhuxianzhen consist of manual carving of patterns and characters on the wooden board and color printing with water and ink. There are different steps in the producing process, including painting, carving, publishing, mounting and so on. The color inks used for the color printing are extracted from traditional Chinese medicinal herbs, and as a result all the printed pictures have extremely bright colors that could last for a long time without fading. Hence Zhuxianzhen New Year woodprint has deep connotation in both technology and art history.

Thirdly, it has support and privilege of developing from the government. A string of policies relate to cultural industry development have been introduced in order to guide and support its developing. More practical strategies and more financial support have been offered by Kaifeng government in order to accelerate the industrialization of Zhuxianzhen New Year woodprint. In March 2006, the declaration of the issue of special stamps on the theme of Zhuxianzhen New Year woodprint was launched officially. In the same year, Kaifeng government provided 1million yuan as initial capital for its development. What's more, a special route for the travelers to visit Zhuxianzhen was founded that year. June 2007, the principles for Zhuxianzhen New Year woodprint developing association were published after a long preparation. On May 6th, an exhibition about Henan culture and folk art was held in the Chinese cultural center in Seoul, in which Zhuxianzhen New Year woodprint, as a national non-material culture heritage, was exhibited and appreciated by Koreans. 


\section{THE DEVELOPING STRATEGIES FOR ZHUXIANZHEN NEW YEAR WOODPRINTS' INDUSTRIALIZATION}

\section{A. Scientific planning and marketizing operation are necessary.}

It is important that the government, with the concept of "to promote business by folk culture; to protect the culture with business", should lead the relative authorities to collect and accumulate Zhuxianzhen New Year woodprints, and start the overall arrangement of its industrialization. The building of Zhuxianzhen New Year woodprint Museum which was started on Dec 28th, 2008, with an investment of 32 million yuan, is divided into three phases. The first phase has been finished. The museum will have the New Year woodprint's origin of hall, ancestor craftsmen hall, displaying hall, high-quality products hall, and exhibition and sales hall.

\section{B. The construction of creative talent team is also important.}

The 12th Five-Year Plan of China shows the government's emphasis on talents. The education and cultivation of creative talents cannot be ignored in the future business of Zhuxianzhen New Year woodprint. Hence, we should try the best to cultivate talents with innovation ideas who can understand culture and are good at marketing at the same time. In addition, we should try to provide a good environment for the talents, in which they can found their own opportunities and development. Their gifts and characteristics should be respected in the environment; creative thinking and bold innovation should be encouraged as well.

\section{The founding of creative industry park is the third developing strategy.}

The key factor in this strategy is the selection of experiment industry park, which should be based on the excellent foundation of economy, society, technology and culture development. In the operation of creative industry park, some feasible new industrial forms can be experimented, for example, the arrangement and setting of New Year picture design department, selling products research department, reading center and tea house. According to the different needs and tastes of possible consumers, new consuming process, including buying, experience and interactive participation, etc. should be advocated and promoted.

D. Its status in the Internet market should be considered as well. The Internet starts the irresistible online shopping trend.

With this backdrop, some researchers believe that the great value of Zhuxianzhen New Year woodprint lies in its "oldness", but the development of it lies in "new" ideas. We must try to refashion it, to bring it new content and energy by the utilization of modern information technology. So it becomes urgent for us to build the selling platform on the Internet, and to explore new marketing channels.

\section{E. Innovation ideas should be used in the marketing system in order to promote the brand value of Zhuxianzhen New Year woodprint.}

According to marketing theories, innovation should be emphasized in the marketing mode for all kinds of products. Zhuxianzhen New Year woodprint is a type of merchandise; however, it is an art first. The best marketing strategy for it is to take the advantages of both of its two features above at the same time. The local government should accentuate and promote the development of its New Year woodprint's industrialization, depending on the positive effects of its historical and cultural background, thus to boost relative industries. In a time of information explosion, the function of advertisements is obvious. The government of Zhuxianzhen and relative authorities should use more effective methods to promote its cultural brand.

\section{F. Another effective strategy is to create a new tourism brand and to accelerate the industry transformation and upgrading.}

Since Zhuxianzhen has its own brand, we should do the utmost to take advantage of the brand effect. The use of History and culture elements can stimulate the tourism industry. By the construction of Zhuxianzhen as a brand for tourism, the transition and improvement of traditional tourism can be achieved. In Zhuxianzhen, there are impressive historical and cultural elements, such as YueFei Temple and his legend, Song Dynasty culture, Muslim culture, New Year woodprint, GuanYu legend and so on. Zhuxianzhen New Year woodprint is still the most potential element. It will bring a new booming time for the whole tourism in the town and the city if the shining points of it can be well promoted.

\section{G. To design and make new products full of innovation is also an effective way to stimulate local economy.}

Nowadays, innovation becomes one of the biggest deciding factors in the process of new products exploration. It is also a key factor in the market adjustment of Zhuxianzhen New Year woodprint. This paper insists that modern elements should be considered about the content and images in New Year pictures, and modern methods should be applied to the production and marketing. The following two paragraphs are about these two points.

\section{CONClusions}

About the content and images in Zhuxianzhen New Year woodprint, innovation ideas should be used. What's more, the shapes, images and colors of Zhuxianzhen's New Year pictures can be applied to the logos of local tourism. Creative tourism products can be explored, such as cultural classic, theme parks, films and programs, cartoon and video games, food, souvenirs and so on.

As for the design of tourism souvenirs, plenty of methods can be used. According to the different themes and situations, design methods vary from each other. We should try to collect the cultural resource in Zhuxianzhen to explore and develop more tourism projects, and to build a comprehensive system there with its historical, cultural and 
folk art characteristics.

Zhuxianzhen New Year woodprint is well-know in China as a leading producer of the woodprint New Year pictures from the ancient times; it is one of the four Chinese New Year woodprints with profound history and distinctive local flavor. Its value can be evaluated from more than one perspective, such as sociology, culture, history, folk customs, aesthetics and so on. It becomes necessary for us to make comprehensive thinking and planning on the industrialization of Zhuxianzhen New Year woodprint, which will be beneficial not only to its protection, but also to local economic development.

\section{REFERENCES}

[1] Daniel J.Marcucci.Landscape history as a planning tool[J].Landscape and Urban Planning, 2002 (49)

[2] Christina von Haren Landscape planning facing the challenge $f$ the development of cultural landscapes[J].Landscape and Urban Planning,2002(60) 